\title{
Multisensor Smart Robot for Border Security Surveillance with Human Action Prediction
}

\author{
N. Vishwanath, S Perumal Sankar
}

\begin{abstract}
Border surveillance (BS) is the most important task in the field of national defense and security. To maintain the peace and to ensure safety of the borders it needs to kept under 24/7 monitoring. Especially, under the current circumstances, like Illegal immigration, importing, implanting explosive device, terrorist activities are common challenges occur in our country border. To curb such happenings on the border areas, the least that can be done is to provide a continuous monitoring. The edge of a country border spreads to several thousand heaps for which human surveillance is more challenge and may lead to loss of human life. To overcome the problem in this paper a new Wireless Multifunctional Smart Robot for Border Security Surveillance with Real Time Object Recognition (OR) system is introduced the proposed robotics system is based on IOT and OR. This method mechanically senses the interruption form the strangers and sends the photos to the admin that categorized which kind of object is to be capture in the image sensor with the help of Navy biases algorithm the Human action has been detected. The multi-sensor Smart robot is proficient for sensing motion using Passive and also Infrared Sensor, poisonous gas using Gas sensor, fire or blast using Flame Sensor, high temperature using Temperature sensor, Camera for capturing the activities in the border, ultrasonic sensor for detecting any obstacles and GPS is used for tracking the location. Any trespasses, bombs, harmful gases, fire and other dangerous situations are sensed and sent to the server. This system detects the dangerous conditions near the border and saves the life immediately without any loss of human life.
\end{abstract}

Key-words: Object Recognition (OR), Raspberry pi, Robotics, Surveillance Monitoring and wireless sensor.

\section{INTRODUCTION}

Security is the level of assurance, which isolates the ownership and potential dangers. Security can be arranged into various sorts; they are data innovation security, physical security, potential security and monitory security and so on [1]. The structure and advancement of robots become a present innovation in our everyday life. Robots are utilized for salvage security and observation [2]. The expertise of robot can be used were a human isn't able for doing it. For observing remote zones a wise robot can be structured with dependable monetary correspondence [3]. This paper presents an Autonomous canny robot for fringe security. A financially savvy independent arrangement is utilized to conquer the restrictions of the current physical arrangements [4]. The quantity of troopers who are selected in the outskirts

Revised Manuscript Received on July 22, 2019.

Dr. N.vishwanath, Professor, Computer science engineering, toc $\mathrm{h}$ institute of science and technology.India.

Dr. S perumal sankar, Professor, Electronics and communication engineering and dean $(\mathrm{pg})$, toc $\mathrm{h}$ institute of science and technology, India. can be diminished. Here the robot can recognize trespassers utilizing video observation, which gives a 24-hour reconnaissance [5]. Interloper discovery is performed with a PIR sensor module connected to the robot. There are fundamentally three areas, to be specific programming segment, equipment segment and Robotic activity. Here a remote control mode is utilized, in which the client explores the robot to the objective area [6].

It has the capacity to naturally identify the trespassers in outskirts and to illuminate close-by client through control unit [7]. Here the robot recognizes hindrances utilizing ultrasonic sensor and catches picture utilizing camera. This empowers powerful ID of flame utilizing fire sensor, distinguishing proof of toxic substances gas utilizing gas sensor, temperature utilizing temperature sensor [8]. Shrewd Robot for Border Security, which distinguishes trespassers utilizing PIR movement sensor, cautions security by catches picture of trespassers utilizing camera in Raspberry pi and alarm the client and it very well may be seen anyplace with the assistance of internet[9]. It empowers security staff to distinguish adequately and easily to recognize an interloper. Plan and Development of Robot vehicle for Border security [10]. The work exhibits a multipurpose savvy robot vehicle utilizing remote camera for recognizing people, fire, metals, obstructions at principle regions and the data is send to fundamental area. The framework uses machine knowledge to give quick reaction from sensors [11].In this paper to improve the reconnaissance security framework a raspberry pi based robots is presented. The proposed mechanical framework comprise of different sensor, for example, inactive infra-red (PIR) sensor which is utilized to distinguish the item. Ultrasonic sensor is utilized to identify hindrances. Gas sensor identifies toxins gas, fire sensor is utilized to identify fire, and temperature sensor is utilized to recognize temperature. The proposed mechanical framework contains one camera, which identifies the human activities.

The rest of paper is organized as follows: Section II surveys several recent papers on Multi-sensor smart robot for border security surveillance. In Section III, the detailed description of the proposed method MSRBSS is presented. In Section IV, the performance of the MSRBSS is evaluated by conducting set of experiments. Finally, the conclusion is made in the Section V.

\section{LITERATURE SURVEY}

In this segment, a study of ongoing procedures in observation security framework with its favorable position, constraints are talked about. In this situation, brief assessments of some 
significant commitments to the current strategies are displayed.

Annu Maria Alex,et al., [12].As innovation increments new dangers and dangers emerge towards national security.

To improve the fringe security, sensor innovation and PC preparing force can be utilized. In our task a self-sufficient shrewd robot is utilized which is upgraded with a video observation camera for identifying the trespasser, advise adjacent control unit and to check whether a gatecrasher is recognized and fire if fundamental. PIR movement sensor is utilized for identifying the trespassers.

Mervin Elsa Jose,et al., [13].proposed a rational framework is required which joins different innovations to make a progressively exact and effective frameworks. Outskirt security is a significant factor worried for some countries. It controls unlawful violations in the nation. By executing an enormous number of robots the security levels can be expanded. A solitary robot could just screen a neighborhood more robots could distinguish interlopers in a worldwide territory. Progressively number of sensors can be incorporated for more security.

SinseeBosch,et al., [14].designed present security strategies to defend our fringes hence usage of android-based robot for outskirt security could be progressively useful. It lessens human association in the fringes. This strategy diminishes the hazard in the lives of our troopers. PIR sensor is utilized to track movement of gatecrasher and camera for video reconnaissance. This framework gives greater security and decreasing the danger of troopers.

ArunKumar,et al., [15].The primary reason for this paper is to depict you how this framework functions, how the innovation which is utilized in this framework works and how it encourages the fighters to verify the outskirts of our nation. This is an electronic framework which is actualized on various fencing areas of outskirt, on the spots where there is no fencing on fringes and where watching of the officers is troublesome or even inconceivable.

DhanajayMishmash,et.al., [16].Implemented the created robot and versatile security framework for the nation fringes. They accept that our framework is exceptionally disentangled and have the highlights that other outskirt security frameworks does not have at versatility level. Our framework can be improved by doing the progressions as indicated by the necessities. The exactness and accuracy of the framework can be improved by utilizing different sensors and it will give great and precise outcomes with high accuracy.

\section{PROPOSED METHODOLOGY}

This methodology is used to develop the current technology and new invention of robots for the border security purpose. The working principles of this method is described below Fig.1.

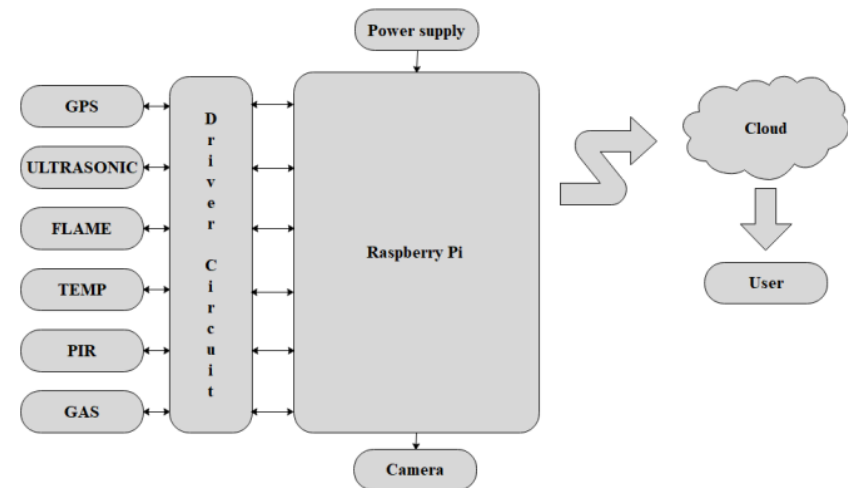

Fig 1. Block diagram of proposed surveillance security system

Working methodology of the Proposed surveillance security systemis to save human in the border area, this system is designed with various sensor such as passive infra-red (PIR) sensor is used to detect the object, ultrasonic sensor is used to detect obstacles, gas sensor detects poisons gas, flame sensor is used to detect fire, temperature sensor is used to detect temperature. To improve the security surveillance system in this paper the new human activity detection system is introduced. Which is working based on the PIR sensor and the camera. Whenever the PIR sensor detects the object at the time the Camera is start working. Whenever the camera detect the human at the time the Activity prediction process has start working. The proposed activity detection has been worked for single object and multi-objects action predictions. The proposed surveillance system can detect the single object activitieslike walking, Skip, Run, Jump and side. The proposed surveillance system can also detect the multi-object activity's likehandshake (hand tremor), kicking, Punching, Pushing, Hugging and Pointing.

\section{a) Passive Infrared Block}

Uninvolved Infrared Sensor is utilized to distinguish the article. PIR sensor get the contribution through warm radiation-it's only warmth of the human body and that warm radiation is convert into voltage and that voltage information is associated with the raspberry pi board. PIR sensor peruses the advanced qualities .Digital information has three stick vcc, information and ground. it check the contribution from the board on the off chance that it recognizes high esteem, at that point there is no article establishes. On the off chance that it demonstrates low advanced worth, at that point there will be an article found. Presently the item is identified this sensor naturally faculties and alarm the client and send the message to the client.

\section{b) Ultrasonic Block}

Ultrasonic sensor for detecting any obstacles. Ultrasonic sensor is externally trigger to generate pulse to identify the distance between the object. It gets trigger pulse from the board and sends the output to the sensor. If it detects any obstacles it alerts the user. After emitting the sound, the ultrasonic sensor will switch to receive mode.

\section{c) Flame Block}


Flame Sensor detect the fire. Flame sensor gets the input from the board and that data is transformed to the sensor. If any fire is near it detects and alert the user.

\section{d) Temperature Block}

Temperature sensor detects the temperature. Were it get the input data from the board and the signal is transformed to sensor .it display the alert to the admin.

\section{e) Gas Block}

Gas sensor detect poisonous gas.it get input data from the board and sends the output data to the sensor .there if any smoke or gas is found it detects and the alert to the user.

\section{f) Location Detection Block}

GPS for tracking the location.it get the input from the board and sends the output data to the sensor. This GPS is used to track the current location .it help us to save the time and save human life.

\section{g) Human Detection Block}

Camera is used to detect the object with the help of this object detection it can capture the image and alert the user. The object detection has follow conventional neural network (CNN).In CNN it has two methods, they are Training \&Testing. In training method it capture the image and that image is under go for training. In testing the trained image are tested and GPS is used to track the location. According to this method we can save the human life.

\section{Human activity recognition (HAR)}

Activity recognition is to automate the analysis of events from videos. Surveillance systems, this module was developed in three parts which includes moving object detection, moving object classification and activity classification. Human activity recognition (HAR) module block diagram is shown in the figure 2 .

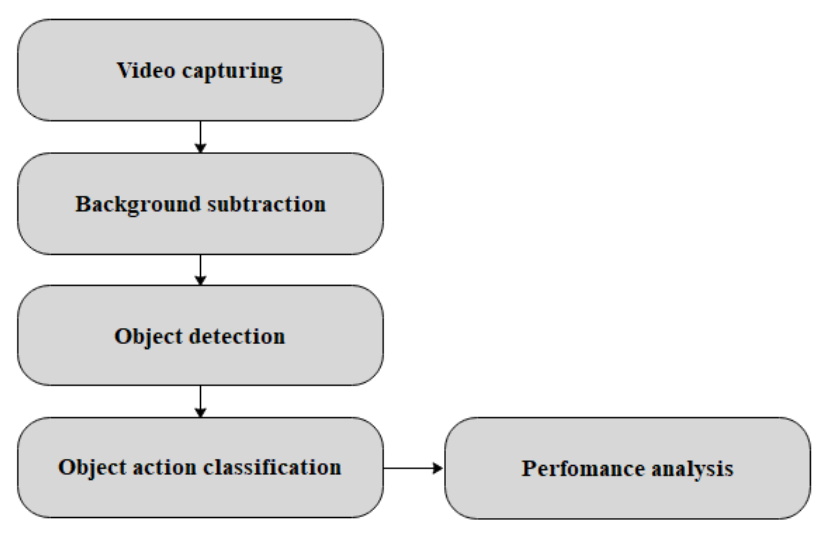

technique was used for classifying actions such as handshake (hand tremor), kicking, Punching, Pushing, Hugging and Pointing. Finally the performance are analysis by using ground truth.

\section{Background subtraction \& object detection}

After the securing of informational indexes, forefront discovery is done on each edge by utilizing foundation subtraction. Here, the foundation of the picture casings is controlled by subtracting the present edge from the past edge or from the normal picture of the quantity of edges. Foundation subtraction functions admirably in explicit states of casing rate and article speed and furthermore it is touchy to the edge. The general equation of foundation subtraction is given in the Eq. (1).

$$
\left|I_{i}(x, y)-I_{i-1}(x, y)\right|>T
$$

Where, $I_{i-1}$ is represented as previous frame, $I_{i}$ is denoted as current frame and $T$ is represented as selected threshold. After foreground detection, blob detection is carried-out for obtaining a particular region of interest to perform further operations like feature extraction, optimization and classification.

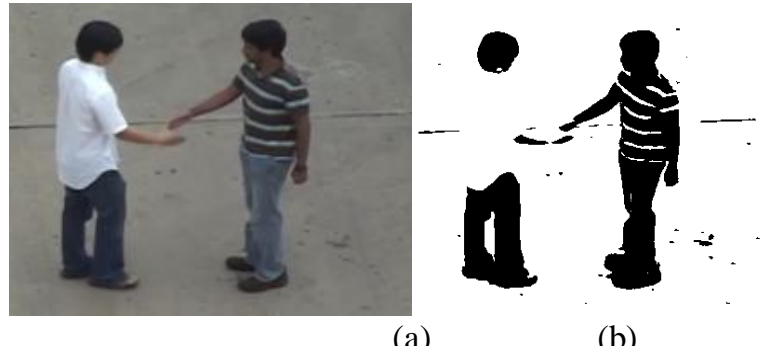

(a)

(b)

Fig.3. a) Input frame, b) Foreground detected frame In the utilization of article recognition or item following, the acquired mass area demonstrates the pieces of items and the nearness of articles. Each mass districts are pressed in level and vertical headings until the whole mass is encased in a square shape box. In this paper, the mass location framework depends on bouncing box, focal point of-mass and nearness pixels. Furthermore, the measurable highlights of masses like volume limited by the enrollment work, area of the middle gravity, pixel tally of the mass, and size of the rectangular fenced in area are likewise decided.

Right now, mass recognition have found progressively prominent, in light of the fact that it uses intrigue focuses for wide gauge stereo coordinating and furthermore for sign ling the nearness of enlightening picture highlights for appearance put together object discovery with respect to the premise of neighborhood picture insights.

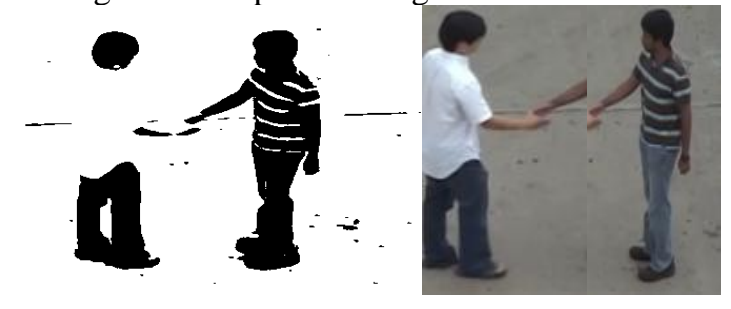

Fig.4. Foreground detected frame and Detection of blobs.

\section{Convolutional Neural networks}

$\mathrm{CNN}$ is the profound learning design and it's generally utilized in numerous PC vision application, for example, picture characterization, sense name ling and so on. Reasonable structure of a picture and progression is perceived. The picture is partitioned into numerous tiles of fixed structure and is nourished into little neural system. Every single removed component is spoken to as highlight map, which is framed by the arrangement of exhibits. From the spectrometer changed over, scale it to $32 \times 32$ picture. The changed over information picture is given to the $\mathrm{CNN}$ layers. In this work $\mathrm{CNN}$

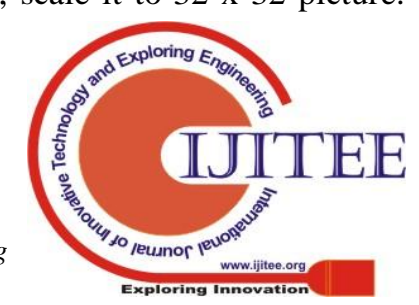


comprise of three Convolutional layers and three Max-surveying layers [28].

The general technique of a Convolution system is to remove basic highlights at a higher goals, and after that convert them into increasingly complex highlights at a coarser goals. The most straightforward was to produce coarser goals is to sub-test a layer by a factor of 2 . This, thus, is an intimation to the convolutions bit's size. The width of the part is picked be fixated on a unit (odd size), to have adequate cover to not lose data (3 would be excessively little with just a single unit cover), however yet to not have repetitive calculation (7 would be excessively huge, with 5 units or over $70 \%$ cover).

A convolution piece of size 5 is appeared in Figure 4. The unfilled circle units compare to the sub sampling and don't should be processed. Cushioning the information (making it bigger so that there are include units fixated on the outskirt) did not improve execution essentially. With no cushioning, a sub sampling of 2 , and a piece size of 5, every convolution layer diminishes the component size from $\mathrm{n}$ to (n-3)/2. Since the underlying MNIST information size $28 \times 28$, the closest esteem which produces a whole number size after 2 layers of convolution is 29x29. After 2 layers of convolution, the element size of $5 \times 5$ is unreasonably little for a third layer of convolution. The primary component layer separates straightforward highlights, which in the wake of preparing look like edge, ink, or crossing point locators. We found that utilizing less than 5 distinct highlights diminished execution, while utilizing more than 5 did not improve it. So also, on the subsequent layer, we found that less than 50 highlights (we attempted 25) diminished execution while more (we attempted 100) did not improve it. These numbers are not basic insofar as there are sufficient highlights to convey the data to the grouping layers (since the portions are $5 \times 5$, we kept the quantities of highlights products of 5). The initial two layers of this neural system can be seen as a trainable component extractor. We presently add a trainable classifier to the element extractor, as 2 completely associated layers (a widespread classifier). The quantity of shrouded units is variable, and it is by shifting this number we control the limit, and the speculation, of the general classifier. For MNIST (10 classes), the ideal limit was come to with 100 concealed units. For Japanese 1 and 2 stroke characters (around 400 classes), the ideal limit was come to with around 200 concealed units, with each other parameter being indistinguishable.

\section{RESULT AND DISCUSSION}

The proposed robotic system is experimented using a raspberry pi. And the simulation testing has been done with the help of desktop computer with 3.0 GHZ Intel i3 processor, 1TB hard disc and 8 GB RAM. For determining the effectiveness of the HSS, the performance of the proposed system is compared with the existing systems on the reputed datasets: UT-Interaction dataset.The hard ware implementation of the proposed System is shown in the below figure

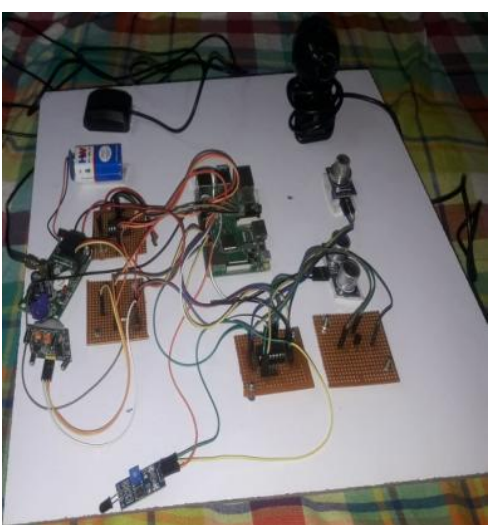

Fig.5. Hardware architecture of proposed system.

\subsection{Performance Analysis}

From the HSS, four unique parameters, for example, True Positive (TP), True Negative (TN), False Positive (FP) and False Negative (FN) are determined. This TP, TN, FP and FN are utilized for computing the presentation. The exhibition parameters broke down in this proposed strategy are portrayed as pursues.

\subsubsection{UT-Interaction dataset}

The UT-Interaction dataset contains recordings of constant executions of 6 classes of human-human communications: shake-hands, point, embrace, push, kick and punch. Ground truth marks for these communications are given, including time interims and jumping boxes. There is a sum of 20 video successions whose lengths are around 1 moment. Some example pictures of UT-Interaction Dataset are appearing in fig.6.
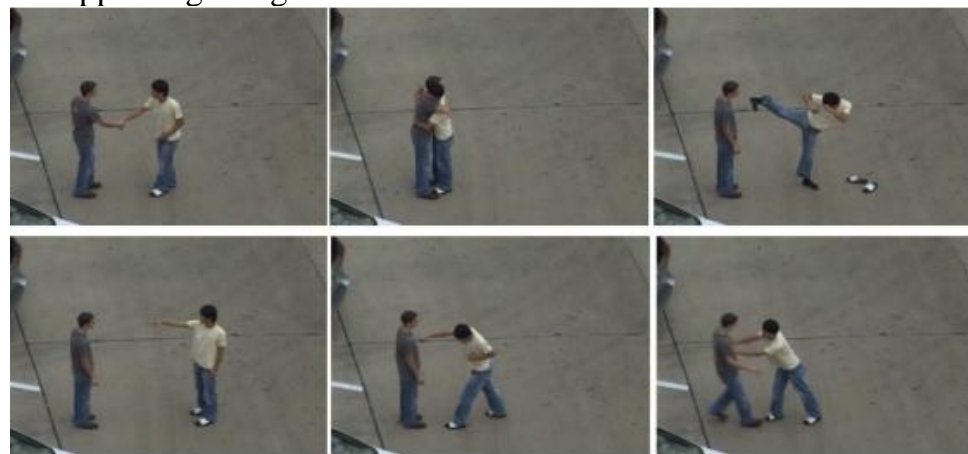

Fig.6. Activities from UT-Interaction Dataset 1: shaking hands, hugging, kicking, pointing, punching and pushing

\section{Recall, Precision}

Recall is the number of True Positives (tp) divided by the number of $(t p)$ and the number of False Negatives $(f n)$. Recall mathematical equations show as (15):

$$
R=\frac{t p}{t p+f n}
$$

\section{Precision}

Precision is the number of tp divided by the number of (tp) and False Positives. It is also called the Positive Predictive (PP) Value. Precision mathematical equations show as (16):

$$
\text { Precision }=\frac{t p+t n}{t p+t n+f p+f n}
$$




\section{Sensitivity}

Sensitivity is a basic property of image processing the. Sensitivity is also called as $t p$ rate. Mathematically, Sensitivity mathematical equations show as (17):

$$
\text { Sensitivity }=\frac{t p}{t p+f n}
$$

\section{Specificity}

The specificity provides, how likely the test is to come back negative characteristic. Specificity also called the true negative rate, Mathematically, Specificity mathematical equations show as (18):

$$
\text { Specificity }=\frac{\mathrm{tn}}{\mathrm{tn}+f \mathrm{fp}}
$$

\section{Accuracy}

By using the Specificity and Sensitivity the Accuracy of the image is calculated. Accurately represent the quantity of the image. Mathematically, mathematical equations show as equation (19):

$$
\text { Accuracy }=\frac{t p+t n}{t p+f p+t n+f n}
$$

\subsection{Quantitative and comparative analysis on} UT-Interaction dataset

In this sub-section, UT-Interaction dataset is assessed for evaluating the performance of the HSS technique. The detection of action in the UT-Interaction dataset is denoted in the Fig.7.
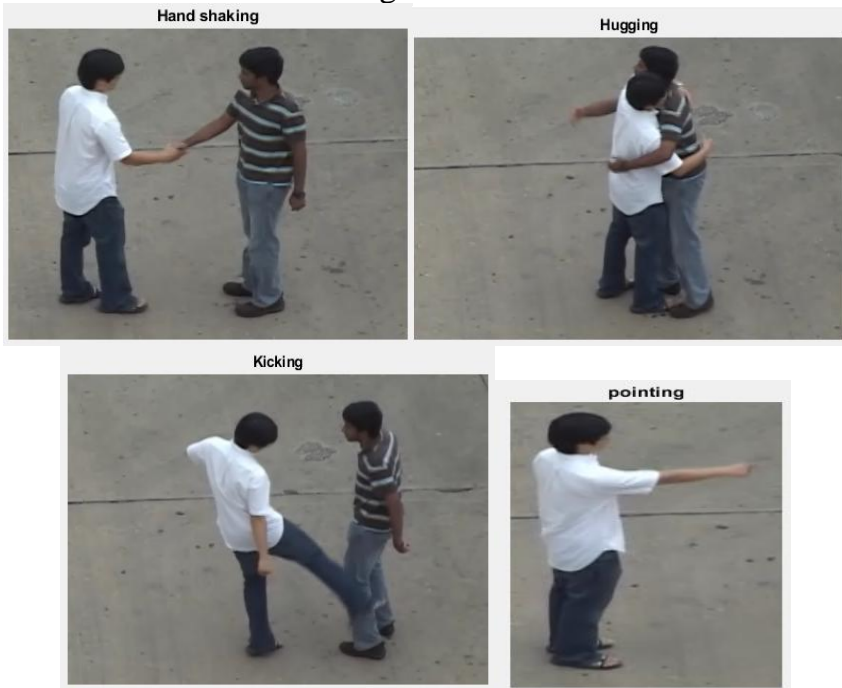
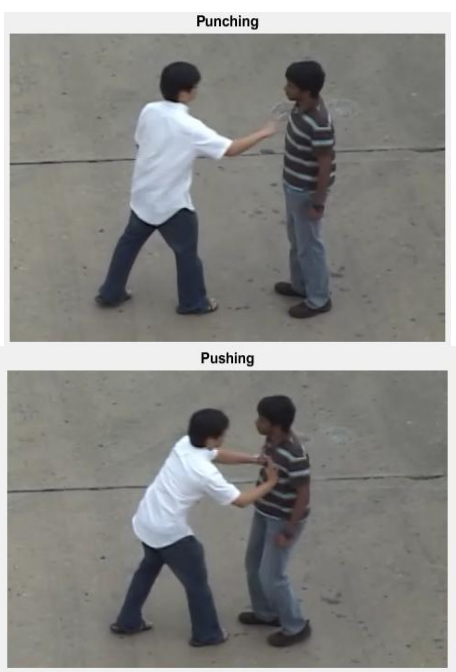

Fig.7. Action detection in the UT-Interaction dataset

Tab.1. Comparative analysis of proposed and existing works in UT-Interaction dataset

\begin{tabular}{|c|c|c|c|}
\hline Year & Method & Dataset & Accuracy \\
\hline 2016 & $\begin{array}{c}\text { Spatio-temporal } \\
\text { features context [23] }\end{array}$ & UT-Interaction & $\mathbf{9 2 . 3 \%}$ \\
\hline 2017 & $\begin{array}{c}\text { Stage model + HOG + HMM } \\
{[24]}\end{array}$ & UT-Interaction & $\mathbf{9 4 \%}$ \\
\hline 2019 & HSS & UT-Interaction & $\mathbf{9 6 . 4 1 \%}$ \\
\hline
\end{tabular}

In Table 1, the HSS technique performance is validated by means of accuracy. Here, the performance evaluation is validated with $80 \%$ of training and $20 \%$ of testing. The proposed system results is an average results of six various actions. From the tabulation 1. And Fig.8. analysis shows that proposed HSS technique is providing much better results compared to the existing systems Spatio-temporal features context [23] and Stage model + HOG + HMM [24].

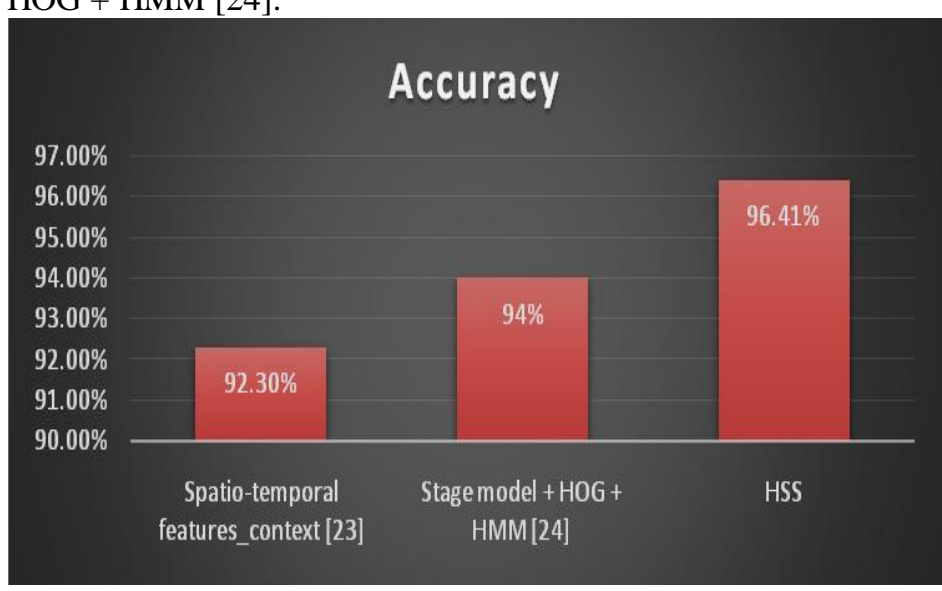

Fig.8.Comparative analysis of proposed and existing works in UT-Interaction dataset.

\section{CONCLUSION}

In this paper, the structure and usage of fringe security observation utilizing robot was actualized the proposed framework clarify the capacity of the robot. With the assistance of cloud, it very well may be gotten to anywhere. it is utilized to screen the fringe

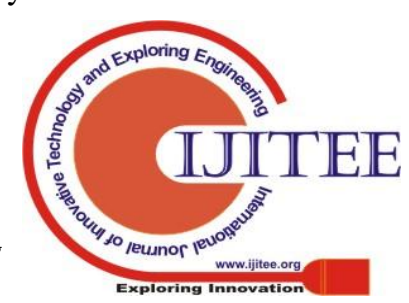


regions with the assistance of remote sensor. The proposed robot framework comprise of such a large number of focal points, for example, Infra-red identification, Ultrasonic recognition, Flame location, Temperature discovery, Gas identification, Human Detection and Weapon Detection. The proposed Human activity location framework has been contrasted and existing frameworks Spatio-worldly includes setting [23] and Stage model + HOG + HMM [24] and with the assistance of UT-Interaction dataset. Contrasted with the current framework the proposed framework gives much better outcomes regarding accuracy. Afuture improvement the framework could be upgraded with reconnaissance at the in-flight.

\section{REFERENCES}

1. Ajith T Alias, MA college of engineering, Kothamangalam."ultrasonic vision for border security"'[2016].

2. Android based intelligent robot for border security [2014], IJISET, Vol.1 issue 5,malanad college of engineering, Karnataka.

3. Study of android operating system and its versions, IJSEAS, Vol.2, Issue-2, February 2016 MPhil scholar, PSG College of Arts \& Science, Coimbatore, India. 4.Study of android operating system and its versions, IJSEAS, Vol.2, Issue-2, February 2016 MPhil scholar, PSG College of Arts \& Science, Coimbatore, India.

4. 5.Minni Mohan, Department of electronics and communication, M.A College of Engineering, Kothamangalam,BorderSecurityRobot,IJCI,vol.5.no.2,April 2016,A P J Abdul Kalam Technological University, Kerala, India.

5. 6. "BorderSense: Border patrol through advanced wireless sensor networks", Zhi Sun, Pu Wang, Mehmet C,Vuran,Mznah A Al-Rodhaan,AbdullahM.Al-Dhelaan,IanF.Akyildiz Ad Hoc Networks 9 pp. 468-477 2015.

6. 7. K. V.S. S. S. S. Sairam, N. Gunasekaran, S. Rama Reddy, "Bluetooth in Wireless Communication", (2012), IEEE

7. 8.Bellazreg, R. et al. (2013) 'Border surveillance: A dynamic deployment scheme for WSN-based solutions', in Wireless and Mobile Networking Conference (WMNC), 2013 6th Joint IFIP, pp. 1-8.

8. 9. Bellazreg, R., Boudriga, N. and An, S. (2013) 'Border surveillance using sensor based thick-lines', in Information Networking (ICOIN), 2013 International Conference on, pp. 221-226.

9. 10.Berrahal, S. et al. (2016) 'Border surveillance monitoring using QuadcopterUAVAided Wireless Sensor Networks'. Udrugazakomunikacijskeiinformacijsketehnologije,

Fakultetelektrotehnike, strojarstvaibrodogradnje, Sveu $\{\check{c}\}$ ili $\{\check{\text { s }}\}$ te u Splitu

10. 11. Sungbok Kim and Hyunbin Kim, "Simple and Complex Obstacle Detection Using an Overlapped Ultrasonic Sensor Ring,"2014 12th International Conference on Control, Automation and Systems

11. 12. Jun Hou, Chengdong Wu, Zhongjia Yuan, Jiyuan Tan, Qiaoqiao Wang and Yun Zhou, "Research of Intelligent Home Security Surveillance System Based on ZigBee," International Symposium on Intelligent Information Technology Application Workshops, pp.554-557,21-22 Dec.2018 .

12. 13. J. Borenstein, H. R. Everett, and L. Feng, "Where Am I?": Sensors and Methods for Mobile Robot Positioning, The University of Michigan, 2016.

13. 14. J. L. Crowley, "World Modelling and Position Estimation for a Mobile Robot Using Ultrasonic Ranging,"Proc. IEEE Int. Conf. Robotics and Automation, pp. 674-680,1989,2017.

14. 15. H. Choset, K. Nagatani, and N. A. Lazar, "The Arc Traversal Median Algorithm: A Geometric Approach to Increase Ultrasonic Sensor Azimuth Accuracy," IEEE Trans. Robotics and Automation, vol. 19, no. 3, pp. 513-522, 2016.

15. 16.F. Porikli, Y. Ivanov and T. Haga, Robot abandoned object detection using dual foregrounds, EURASIP Journal on Advances in Signal Processing, (2018) article no. 30.

\section{AUTHORS PROFILE}

Dr. N.vishwanath, professor, Computer science engineering, toc $\mathrm{h}$ institute of science and technology.

Dr. S perumal sankar., Electronics and communication engineering and dean(pg), toc $\mathrm{h}$ institute of science and technology. 